\title{
EDITORIAL
}

\section{Impressions of the Indiana Global Health Research Conference: An Editor's Account}

\author{
Jeffrey L. Jackson, MD, MPH \\ C, GIM Section, Zablocki VAMC, Medical College of Wisconsin, Milwaukee, WI, USA.
}

J Gen Intern Med 28(Suppl 3):S621-4

DOI: $10.1007 / \mathrm{s} 11606-013-2454-9$

(C) Society of General Internal Medicine 2013

This editorial is informed by participation in the conference, review of the supplement articles and interviews with several conference attendees. Where applicable, quotations are included in quotes and are italicized.

As the editor for this supplement, I drove down from Milwaukee to the meeting in Turkey Run State Park, located west of Indianapolis, and participated in the small groups and conducted interviews with various participants. Interviewees were either nominated by the meeting organizers, were presenters at the meeting or were selected by happenstance during the process of attending the meeting. As editor, I also reviewed all the supplement articles. I am not personally involved in global health research and came to the meeting with mixed feelings. On the one hand, I fully understand the allure of "going far foreign and seeing new countries." After residency, my initial 3-year tour of duty as an Army Captain was in Nuremburg, Germany. Later, while stationed at Walter Reed, I frequently served as "trip doctor" for congressional delegations, and whenever possible, tried to speak with local providers about their healthcare systems. I've published articles contrasting the American healthcare system with the German and Dutch systems, and have served as visiting Professor to the Otowa Clinic in Kyoto Japan on two different occasions. In these travels, I have seen a wide range of healthcare systems, from extremely high-functioning to extremely dysfunctional ones. The disparity in health and healthcare systems between resource-poor and resource-rich countries is striking; the need and opportunity to make a significant and lasting difference is real. The instinct to serve is strong in many physicians, myself included. On the other hand, a part of me is honestly isolationistic and even embarrassed about U.S. interventions in health internationally. How can American healthcare organizations justify trying to improve health care in other countries when there is no shortage of problems and

Published online June 25, 2013 disparities here in the United States? In advance of the meeting I was also concerned that, however wellintended, interference by western organizations in lowincome countries could have unintended consequences. In one of my own expeditions abroad, providers in Rwanda had complained that there was money for "sexy" diseases, such as HIV, but no money for other important problems such as diabetes and hypertension. They felt the care they provided and their healthcare system was being distorted by well-meaning international organizations. To be frank, then, I attended the meeting with some predispositions to contrarian notions, personal biases, and frank skepticism. Perhaps as a consequence, I challenged each person I interviewed with tough questions.

An initial question asked of all interviewees regarded their personal motivation to be involved in global health. Perhaps not surprisingly, there was some reticence to speak of this, perhaps born of a reluctance to speak of "self." In the end, however, all respondents observed that upon reflection, medicine is, at its essence, altruistic and should be based on commitment to service. They all reported being strongly motivated by a desire to "do good" and to serve one's fellow human beings. They also echoed my feelings of feeling appalled at the extreme disparities in health care between resource-rich and resource-poor countries. Many spoke of prior experiences in the Peace Corps or in missionary work. Service to mankind was a universal motivator among my interviewees. "What really grabbed me with this idea was that in these incredibly underserved and impoverished populations, with the right kind of intervention you could really make a huge difference in the lives of larger groups of people. Something as simple as a literacy program for women and children actually changes lives." Other motivations were a commitment to cross-cultural environments, health development as peacemaking, and recognition that some specific scientific resources (e.g. genomic diversity, certain endemic diseases) are present in LMIC (Low and Middle Income Country) environments.

Another tough question was how they could justify working on global health issues when there were so many problems at home. "Before we started thinking about primary care in a global sense, we worked hard to provide 
good networks of primary care for all people in Indianapolis. We felt strongly that it would be hypocritical to provide care internationally when there was an unmet need in our own backyard." A pediatric oncologist, when asked about developing children's oncology treatment groups in Kenya, when those same children lacked access to clean water, immunizations and other basic public health, responded: "For me, pediatric cancer is a relatively simple ethical situation. Most of the childhood cancers are not only curable, but treatable with generic chemotherapy. Treatment is for a finite cost, for a finite period of time, after which you return someone to society who can work and contribute for a life-time. It is a much simpler ethical decision than the one to spend an enormous amount of limited resources on incurable diseases, such as HIV."

My own concern about distorting local health care systems was not shared by others, rather the opposite. Most, when asked, were more concerned with having no lasting impact than on permanently distorting healthcare systems. As a Kenyan doctor said, "Outside organizations, though well-meaning, have the potential to distort the health care system. But a bigger problem is lack of sustainability. While the initial idea may be very good, the sustainability of the project is not always factored in." Another (North American) provider said, "I do worry some about [distorting healthcare systems], but these countries are resourceful and are going to take advantage of what they can get. Ultimately, that's a decision that each country has to analyze and prioritize and figure out how they're going to allocate the limited resources they have. In the meantime, they take advantages of any opportunities they can get. I actually worry less about distorting their healthcare system than not having any long-lasting impact."

Working for sustainability was highlighted by all interviewees as a deep concern and a foundational principle in global health engagement. "If you give a man a fish, he eats for a day. If you teach a man to fish, he eats for a lifetime. Academic medical centers are uniquely poised for this mission. They have a tripartite mission: clinical care, education and research. [In our program] Every dollar we spend is a dollar spent on all three components. We provide clinical care, while training local providers. We both study our outcomes and teach the next generation to study their outcomes."

Several components of an activity were felt to contribute to sustainability. "There's a long list of failed projects and I assume that they were all initiated by people who had the best intentions, but they failed because of lack of connection to or lack of engagement with the affected community. I think number one is gaining the trust and having a two-way relationship with the people you're working with...We're going to learn from them every bit as they're going to learn from us. We have to go into it with that kind of understanding and be sincere about that." Community engagement was a consistent theme. "From the beginning the community must have input, the community benefitting from the program must be given control." Another person said, "You have to really understand the local circumstances. For example, with water in Africa, the main players are women and children. If you are going to make water projects work in Africa, you have to engage them. You have to give the local community control of the project. They have to take ownership and make the critical decisions, they have to make the decision that the project is important, they have to make a tangible investment, in resources or in human capital, for it to be sustainable." To be sustainable, the local population needs to be in control, to be able to set priorities and to develop projects and systems that work within the constructs of local moral and cultural customs and resources.

A concrete example of understanding local mores and problems with applying Western cultural norms is the use and management of financial leverage. "One has to be careful about money in African countries. In Kenya, you're not just feeding yourself; you're feeding your tribe. A common African phrase, [now a book title], is "It's Our Turn to Eat." It reflects a common belief in African culture...you don't just eat 10 or $30 \%$, you eat $100 \%$; sometimes meaning you steal $100 \%$ and hope they'll give you another large amount of money. The World Bank has funded a road to the Maasai Mara three times and never a piece of pavement was laid. A well-known US researcher arrived in Kenya with over a million dollars in research support. He left a year later with nothing to show for the money spent. Worse than the million and a half bucks wasted, the US researcher came in and sucked the best and the brightest right out of the classroom, right out of the clinics and plunked them down into a research project, and distorted what gets prestige, what gets money, what gets elevated... When we first got to Kenya, we purposefully didn't spend a lot of money. We just showed up and participated. We took care of patients and we helped with medical education. We knew we needed to learn from them what they needed and how they needed our help."

Another important principle that was cited is the need to be keenly aware of the possibility of unintended consequences. One interviewee spoke, for example, of an American program to import free bicycles to Rwanda, noting quite rightly that most of the population relied on walking to get anywhere and bicycles could be a source of inexpensive, sustainable, improved transportation. Alas, the free bicycles had driven the local entrepreneurs out of business and many of the bicycles wound up in the hands of local criminals who sold them on the international market at a discounted price.

Also highlighted by many was the need to measure the impact of interventions and to conduct basic research. 
"Many countries, such as Kenya are geographically huge, and resource-poor. Often it is unclear exactly what the health problems are, making it difficult to know how to intervene. What is the prevalence of diabetes or hypertension? No one knows. The functionality of any intervention is going to depend on the local health care capacity and the resources available."

Many of the interviewees were concerned about trying to export the worst of our practices. Speaking of the U.S. Health research enterprise, one participant said, "We spend a billion dollars a year. The ideal researcher has several NIH RO1 grants with numerous post-docs working on narrower and narrower medical questions; most of which have little to no chance of impacting health care or health care outcomes. If this talent were focused differently, the dividends could be enormous." Another participant spoke with disdain about a project to instill a state-of-the-art cardiac catheterization lab in Nairobi, the capitol city of Kenya. "Here is a country lacking in basic medical care, with little to no primary prevention such as hypertension screening or treatment, tobacco cessation, lipid control. Smoking is rampant in Africa. Yet our answer is to put in a cardiac catheterization lab. This doesn't strike me as a high priority project for a resource-poor country."

Other important lessons from participants and their contributed papers in the conference included:

1) The need to give careful attention to the ethics of conducting global research. While some concepts of research ethics may be universal, the process of interpreting and applying these standards should be locally situated and controlled. The Belmont report, developed in the U.S., emphasizes respect for persons, justice and beneficence. But the concept of "person" is a culture-bound notion. Some ethnic groups are centered on clan, tribe or community rather than the individual. Concepts such as justice and beneficence also are strongly culturally normed. For example, in North America, it is unthinkable that consent for a woman to participate in research would - as a matter of routine - be provided by the husband, while it is possible that this might be seen as logical in certain traditional cultures. Researchers involved in global research need to understand the local cultural forces that shape policy and research decision-making.

2) The economics of health care and health care delivery, innovations that enhance cost-effectiveness globally, are especially worthy of research in resource-scarce environments. The lessons from health care delivery models that are emerging in other countries are a potential source for identifying potential best practices. What is learned in resource-scarce environments might be relevant both locally and in the northern hemisphere. Resource-poor countries are often forced to innovate and streamline health care processes to maximize efficiencies, and findings there can inform best practices in resource-rich countries. Task shifting and lay health workers that radically alter primary care are more likely to be demonstrated in LMICs than in the West. As resource-poor countries develop health care delivery systems, they can, hopefully, learn from the mistakes of resource-rich countries and avoid wasteful health care spending; dollars spent that often achieve only minimally improved or even worse health outcomes. Unfortunately, there is little evidence of this happening. With scarce resources, LMICs need to focus on essential health services, core national formularies and essential technologies. At best, they are innovating from the bottom up. Where they go wrong, it's in the desire to emulate the resource rich environments and develop highly specialized services that become "black holes" for national resources, both financial and human.

3) The capacity of laboratory support, both for clinical and research, varies widely, but there are typical patterns of development and organization. Understanding how laboratories operate - the constraints and limitations and opportunities - is important for engaging in meaningful global health projects.

4) It may be possible, through development of good electronic medical records (EMR), data-basing and point of care sophisticated computer algorithms, to predict an individual patient's predisposition to disease and prognosis to a greater extent than currently possible. Research focused in this area may lead to better allocation of health resources for prevention and treatment. This may be possible even in resource-poor countries, if they develop standard, nation-wide EMR programs as they grow their health programs. Ironically, informatics data-mining and "personalized" medicine may emerge in LMICs more rapidly than in some developed countries.

5) Other examples of 'reverse-innovation' (from LMICs in the South to the developed economies in the North) may involve electronic medical records themselves. Open-source EMRs like 'OpenMRS' were developed for Africa, but are rapidly disseminating. OpenMRS is now the most widely used EMR on the globe.

As I drove home, I felt considerably more optimistic. None of my "radical" thoughts were truly contrarian after all. All the individuals I had interviewed were thoughtful about their involvement in Africa and in their potential impact, both intended and unintended. Interviewee voices echoed in my head, "Our whole pediatric research program was built based on needs assessment by the Kenyan pediatricians of what we would tackle and what made the most sense to focus on priority wise." "The other thing to judge by is what you leave behind. Through our partnership, we've built a health care system upon which we can address all manner of health 
challenges for people in Kenya. While it started out focused as an effort to identify and treat patients with HIV, it has now grown into an infrastructure from which we now can address other chronic diseases, on which we can address primary care needs." "I'm optimistic in terms of the young Kenyans, of the bright, young medical students and residents coming up who are so dedicated and are willing to put up with so much that we just wouldn't." My only remaining question was whether they'd have any interest in hiring an older, 'cynical,' primary care provider.

Conflict of Interest: The author declares no conflict of interest with any of the material in this paper.
Disclaimer: The views presented in this manuscript represent those of the author and should not be construed to reflect, in any way, those of the Department of Veteran Affairs or the US Government.

Corresponding Author: Jeffrey L. Jackson, MD, MPH; C, GIM Section, Zablocki VAMC, Medical College of Wisconsin, Milwaukee, WI 53295, USA (e-mail: jjackson@mcw.edu).

\section{REFERENCES}

1. O'Bryan P. The Thirteen Gun Salute. NY: W.N.Norton Publishing; 1989.

2. Wrong M. It's Our Turn to Eat: The Story of a Kenyan Whistle-Blower. NY: Harper Collins Publishers; 2010. 